\title{
Desigualdad educacional y socioeconómica como determinante de mortalidad en Chile: análisis de sobrevida en la cohorte del proyecto San Francisco
}

\author{
Elard Koch ${ }^{1 a}$, Tomás Romero², Leopoldo Manríquez ${ }^{3}$, \\ Mario Paredes ${ }^{4}$, Esteban 0 rtúzar ${ }^{16}$, Alan Taylor 4 , \\ Carolinne Román ${ }^{4}$, Aída Kirschbaum¹, Carlos Díaz ${ }^{1 a}$. \\ Socioeconomic and educational \\ inequities as independent predictors \\ for mortality in a developing country. \\ A cohort study in San Francisco, Chile
}

Background: The socioeconomic position (SEP) and educational level of individuals have an inverse correlation with mortality in developed societies. Aim: To assess in a society undergoing a socioeconomic transition, the mortality risk associated to a low SEP (combination of education and income, scale 0-25 points, reference $\geq 10$ points) and low education (education years, reference $\geq 8$ years), adjusting for other known risk factors. Material and methods: In this prospective cohort study, a random sample of 920 subjects, living in San Francisco de Mostazal, Chile, aged more than 20 years (395 males) was examined for the first time in 1997-1999 and re-examined in 2005-2006. All had information about economic household income and level of education. A Cox regression model was used to evaluate the association between mortality and socioeconomic measures. Results: The crude mortality hazard ratio (HR) was 3.34 (95\% confidence interval (CI) 2.88-3.87) and 6.05 (95\% CI 5.04-7.26) for low SEP and low educational level, respectively. After adjusting for age, gender, hypertension, diabetes, dyslipidemia, abdominal obesity, smoking, alcohol intake and family history of cardiovascular disease, the figures were 1.23 (95\% CI 1.04-1.43) and 1.54 (95\% CI 1.23-1.85) for low SEP and low educational level, respectively. Conclusions: In a society in socioeconomic transition, low SEP and especially low educational level are risk factors for mortality even after adjusting for known mortality risk factors (Rev Méd Chile 2007; 135: 1370-9).

(Key words: Developing countries, Education, Socioeconomic Factors, Risk Factors, Mortality)

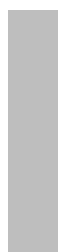

Recibido el 12 de enero, 2007. Aceptado el 11 de mayo, 2007.

Financiamiento: Research Grant FA2005, "Fundación Araucaria”, San Diego, California.

${ }^{1}$ Programa Doctorado, Escuela de Salud Pública, Facultad de Medicina, Universidad de Chile. ${ }^{2}$ Cardiology Services and Cardiac Catheterization Laboratory, Sharp CV Medical Center, San Diego, California. ${ }^{3}$ Unidad de Cardiología, Departamento de Medicina Interna, Hospital Regional de Rancagua, Chile. ${ }^{4}$ Consultorio San Francisco de Mostazal, Chile.

a MPH, alumno doctorado en Salud Pública.

${ }^{\mathrm{b}} \mathrm{MPH}$

Correspondencia a: Elard Koch. Programa de Doctorado, Escuela de Salud Pública, Facultad de Medicina, Universidad de Chile. Independencia 939, Santiago, Chile. Casilla 70012. Teléfono: 56-02-978-6546. Fax: 56-02-737-7121. E mail: ekoch@med.uchile.cl 
$\mathrm{L}$ a desigualdad en el acceso a la salud es un tema de creciente prioridad en numerosos centros de investigación alrededor del mundo ${ }^{1-4}$. En las últimas décadas, investigadores de diversos países han mostrado un incremento de las disparidades en la distribución de la riqueza y en la salud pública global ${ }^{5}$. Aunque Chile ha experimentado un crecimiento económico sostenido, el informe de Desarrollo Humano de las Naciones Unidas 2005 lo posiciona como el undécimo país más desigual del mundo ${ }^{6}$. El 10\% de los hogares más ricos recibe $47 \%$ de los ingresos totales del país. De acuerdo con estudios de Torche ${ }^{7}$, además existiría una barrera para la movilidad entre los sectores de mayores ingresos y el resto del grupo social, que pareciera depender en gran parte de la capacidad de los primeros de financiar una educación de calidad superior a sus hijos, al que los sectores más pobres no alcanzan ${ }^{8}$.

De acuerdo con la hipótesis de la desigualdad del ingreso basada en los trabajos de Preston ${ }^{9}$ y Rodgers $^{10}$, la expectativa de vida individual dependerá del ingreso económico y de la distribución del ingreso dentro de la comunidad en la que el individuo reside. Por otra parte, estudios epidemiológicos longitudinales en Europa y Estados Unidos de Norteamérica (EE.UU.), muestran que el nivel de educación, un indicador frecuentemente utilizado para definir la posición socioeconómica individual ${ }^{11}$, presenta una fuerte relación inversa con la mortalidad por cualquier causa, por enfermedades crónicas y por causas externas ${ }^{12-19}$.

En Chile, indicadores como tasas de natalidad, mortalidad general, mortalidad materno-infantil, índice de fertilidad y expectativa de vida, son comparables a las cifras de países desarrollados. Asimismo, las enfermedades cardiovasculares y los tumores malignos emergen como las principales causas de muerte en la población ${ }^{20-22}$. En este escenario, estudios transversales y ecológicos han establecido un gradiente socioeconómico sobre la presencia de factores de riesgo, mortalidad ${ }^{23-27}$ y percepción de salud en la población ${ }^{28-29}$. En este estudio, evaluamos la hipótesis de la desigualdad socioeconómica y educacional como un determinante de mortalidad general por enfermedades crónicas no transmisibles (ECNT), independiente de otros factores de riesgo convencionales, a través de un análisis de sobrevida en la cohorte del proyecto San Francisco (PSF) ${ }^{30}$.

\section{MATERIAL Y MÉTODO}

La investigación está basada en datos provenientes del estudio longitudinal conducido en el pueblo de San Francisco de Mostazal (13.055 habitantes $>15$ años, censo 2002), que comprende una muestra aleatoria simple de 920 personas (>20 años, 395 hombres) residentes en la zona urbana. Todos los participantes fueron examinados entre 1997 y 1999. El detalle del muestreo de línea basal, el cálculo de tamaño muestral y las variables recopiladas en el PSF han sido descritos detalladamente en publicaciones recientes ${ }^{26,30}$.

Brevemente, se obtuvo información a través de encuestas aplicadas en el hogar, exámenes médicos periódicos y pruebas de laboratorio en el consultorio municipal de San Francisco de Mostazal. Para el diagnóstico de hipertensión arterial, se realizaron mediciones seriadas de presión sistólica y diastólica según guías internacionales ${ }^{31}$. La clasificación de dislipidemia se realizó según los criterios definidos por el ATP III $^{32}$. El diagnóstico de diabetes se realizó siguiendo las recomendaciones internacionales utilizando prueba de tolerancia a la glucosa ${ }^{33}$. Se evaluó consumo de tabaco considerando cantidad de cigarrillos fumados por día y consumo de alcohol aplicando el cuestionario $\mathrm{EBBA}^{34}$. La obesidad abdominal fue definida por la razón entre cintura y estatura ( $\mathrm{RCE}>0,55$, ambos sexos) que ha mostrado un mejor valor predictivo para eventos cardiovasculares no fatales ${ }^{35}$ y una mejor relación sensibilidad-especificidad para detectar factores de riesgo metabólico ${ }^{36}$.

El nivel de educación (NEd) se obtuvo por autorreporte en la encuesta de línea basal aplicada a cada participante a través de los años de escolaridad aprobados, dicotimizada en NEd $<8$ años de escolaridad formal, que es utilizado para definir educación básica incompleta por el Ministerio de Planificación de Chile (MIDEPLAN). Para clasificar el nivel socioeconómico (NSE) de cada individuo, se consideró una escala de puntuación (0-25 puntos) que relaciona los años de educación alcanzados y el ingreso monetario del hogar transformado y actualizado a número de ingresos mínimos de acuerdo con el valor fijado por el MIDEPLAN. Cada año de educación aporta un punto, con máximo de 15 y cada sueldo mínimo aporta otro punto con máximo de 10. Para análisis subsecuente, la variable se dicotomizó en 10 
puntos de acuerdo con estudios recientes en los que se corrobora una asociación de este punto de corte con la presencia de factores de riesgo cardiovascular ${ }^{30,35}$.

En comunicaciones previas se ha mostrado que la cohorte del PSF posee una composición estrechamente similar a la distribución real de la población ${ }^{30}$ y prevalencias similares a la Encuesta Nacional de Salud ${ }^{38}$. En el presente estudio, todo el análisis estadístico fue realizado incorporando pesos censales de acuerdo a sexo y edad (Weighted Sample Analysis, WSA) según la metodología descrita por Barroso Utra et $a^{37}$ con el objeto de obtener estimadores insesgados por error de muestreo aleatorio y corregir diferencias de proprocionalidad entre población muestral y real. Para el cálculo de los pesos individuales se consideraron datos del censo 2002, los que fueron ingresados al programa SPSS 13.0.

En la línea basal se calcularon prevalencias y odds ratio (OR) para evaluar asociación con factores de riesgo. Las medias estadísticas fueron comparadas utilizando ANOVA asumiendo la homogeneidad de varianzas evaluadas a través de test de Levene. Durante el seguimiento se estableció como "falla", la muerte corroborada en primera instancia por certificado de defunción. Para la clasificación final de las causas de muerte, el PSF utiliza el CIE-10 junto con un estudio de cadena causal basado en el seguimiento del registro clínico de cada participante ${ }^{30}$. Este procedimiento permite minimizar el sesgo de selección por mala clasificación diferencial y excluir causas de muerte no relacionadas con ECNT, es decir, enfermedades exclusivamente infecciosas y causas externas que no forman parte de los desenlaces estudiados en este análisis. El número total de años-persona expuesto a riesgo fue calculado entre los individuos que permanecieron vivos desde el término de los exámenes en la línea basal (1997-1999) hasta el 30 de enero de 2006. El seguimiento de cada participante sólo comenzó cuando éste completó el estudio de línea basal, de manera que los tiempos de inicio individuales fueron variables. Así, se estimaron tasas de mortalidad y densidades de incidencia DI considerando en el denominador la sumatoria de los tiempos $t$ de observación individual. Los respectivos intervalos de confianza al 95\% se estimaron asumiendo una distribución de Poisson.
En un estudio exploratorio, detectamos que existe una fuerte correlación entre el ingreso monetario y años de educación en la cohorte ( $\mathrm{R}$ $=0,50 ; \mathrm{p}<0,001)$. Por esta razón, la función de sobrevida fue calculada usando el método de Kaplan-Meier construyendo curvas separadas para las variables NSE y NEd dicotomizadas. La hipótesis nula de no diferencia entre curvas, fue evaluada a través de test log rank considerando un $\mathrm{p}$-value $<0,01$. El riesgo relativo o hazard ratio (HR), fue estimado usando el método de regresión de riesgos proporcionales de Cox mediante dos modelos por separado, uno para NSE y otro para NEd en cuatro bloques de variables aditivas: 1) HR crudo; 2) HR ajustado por sexo; 3) HR ajustado por edad y 4) HR ajustado adicionalmente por la presencia de otros factores de riesgo para ECNT.

\section{Resultados}

En la línea basal, la población con bajo NSE y bajo NEd presentó mayores prevalencias de factores de riesgo, excepto tabaquismo. En general, la fuerza de asociación basada en la magnitud de los OR observados fue mayor para bajo NEd (Tabla 1). La edad avanzada fue el factor más fuertemente asociado a baja escolaridad. Congruente con esta observación, la media de años de educación formal disminuye linealmente con la edad en ambos sexos ( $\mathrm{p}<0,001$ para la tendencia), observándose que la cohorte menor de 40 años posee 10 años promedio de escolaridad, mientras la cohorte de 70 años o más presenta 4 años promedio de escolaridad (Figura 1).

Del total de 920 individuos (edad 39,5 $\pm 16,3$ años) la pérdida de seguimiento alcanzó a 1,48\% (13 sujetos) durante un tiempo promedio de 8 años. En este periodo se produjeron 47 casos fatales por ECNT. La mortalidad ponderada por los pesos muestrales fue 53,9 por mil en hombres y 48,6 por mil en mujeres, promedio de edad 73,8 años (39-93 años) y 71,5 años (rango 33-99 años), respectivamente. Entre los fallecidos, la media estadística de años de educación fue menor para el sexo femenino (3,5 vs 5,1 años; $p<0,001$ ) y también para la puntuación de NSE (7,4 vs 9,1 pts.; $\mathrm{p}<0,001$ ).

Las DI se muestran en la Tabla 2. El riesgo absoluto fue de 6,0 para la cohorte con menos de 8 
Tabla 1. Prevalencia de factores de riesgo de acuerdo a nivel socioeconómico (N SE) y nivel de educación (N Ed) en línea basal de 920 participantes en la cohorte de San Francisco de M ostazal, C hile

\begin{tabular}{|c|c|c|c|c|c|c|}
\hline \multirow{2}{*}{ Factores de riesgo } & \multirow{2}{*}{\multicolumn{3}{|c|}{$\begin{array}{c}\text { Nivel Socioeconómico (NSE) } \\
\text { NSE }<10 \text { pts. NSE } \geq 10 \text { pts. Odds Ratio (IC95\%)* }\end{array}$}} & \multicolumn{3}{|c|}{ Nivel de Educación (NEd) } \\
\hline & & & & NEd $<8$ años & NSE $\geq 8$ años & Odds Ratio (IC95\%)* \\
\hline Mujer & $53,3 \%$ & $45,6 \%$ & $1,36(1,27-1,46)^{* *}$ & $50,9 \%$ & $47,0 \%$ & $1,17(1,09-1,25)^{* *}$ \\
\hline Edad > 65 años & $17,5 \%$ & $4,9 \%$ & $4,14(3,65-4,69)^{* *}$ & $19,9 \%$ & $2,7 \%$ & $8,85(7,59-10,3)^{* *}$ \\
\hline Antecedentes familiares ${ }^{\dagger}$ & $46,1 \%$ & $41,5 \%$ & $1,21(1,12-1,29)^{* *}$ & $50,2 \%$ & $38,6 \%$ & $1,60(1,49-1,72)^{* *}$ \\
\hline Hipertensión arterial & $32,6 \%$ & $27,7 \%$ & $1,27(1,17-1,37)^{* *}$ & $39,3 \%$ & $22,9 \%$ & $2,18(2,02-2,35)^{* *}$ \\
\hline Diabetes mellitus II & $6,7 \%$ & $3,5 \%$ & $2,01(1,71-2,36)^{* *}$ & $7,3 \%$ & $3,0 \%$ & $2,53(2,14-2,99)^{* * *}$ \\
\hline Dislipidemia ${ }^{\ddagger}$ & $48,8 \%$ & $43,1 \%$ & $1,24(1,16-1,33)^{* *}$ & $55,9 \%$ & $37,7 \%$ & $2,09(1,95-2,25)^{* *}$ \\
\hline Obesidad abdominal $^{\#}$ & $59,1 \%$ & $49,7 \%$ & $1,46(1,36-1,57)^{* *}$ & $67,4 \%$ & $43,6 \%$ & $2,67(2,48-2,87)^{* *}$ \\
\hline Tabaquismo & $26,9 \%$ & $30,0 \%$ & $0,86(0,79-0,93)^{* *}$ & $27,5 \%$ & $29,8 \%$ & $0,89(0,82-0,96)^{* *}$ \\
\hline Consumo de alcohol ${ }^{¥}$ & $31,0 \%$ & $21,4 \%$ & $1,65(1,52-1,78)^{* *}$ & $27,1 \%$ & $23,7 \%$ & $1,20(1,11-1,30)^{* *}$ \\
\hline
\end{tabular}

† Familiar directo muerto por evento cardiovascular; ${ }^{\ddagger}$ Criterio ATP III [ref. 32]; ${ }^{*}$ RCE > 0,55 ambos sexos (ref. 36); ${ }^{\circledR}$ Fumador diario sin considerar cantidad; ${ }^{¥}$ test de EBBA positivo.

* Odds Ratio de prevalencia para un NSE $<10$ puntos y NEd $<8$ años cursados y aprobados.

${ }^{* *} \mathrm{p}<0,01$ para prueba de asociación $\chi^{2}$.

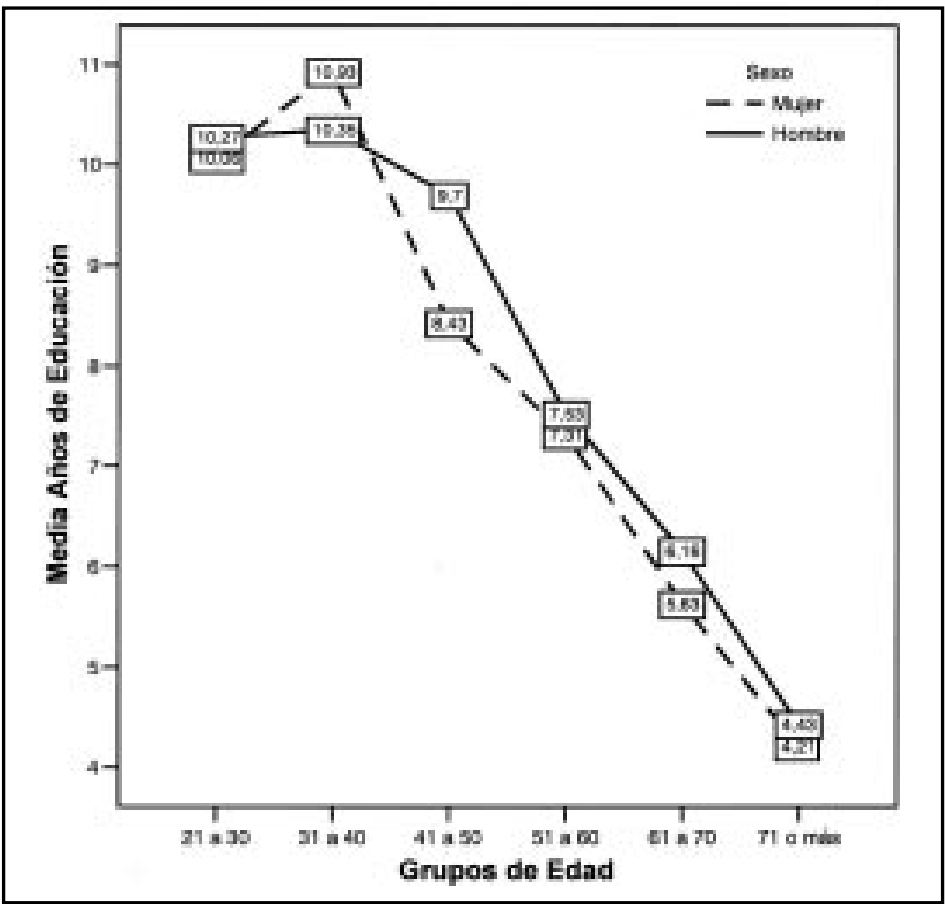

Figura 1. Años de educación promedio de acuerdo a edad y sexo en la cohorte de San Francisco de Mostazal, Chile.

años de educación y de 2,9 para la cohorte con NSE $<10$ puntos. Las curvas de Kaplan-Meier comparando las funciones de sobrevida de acuerdo a NEd y NSE se presentan en la Figura 2. Durante 7.255 añospersona de seguimiento la sobrevida para la cohorte con bajo NEd fue de $89,3 \%$ vs $98,2 \%$ para el NEd alto (log-rank 485,6; p <0,001). Para bajo NSE, la sobrevida fue de $91,1 \%$ vs $97,1 \%$ para el nivel alto (log-rank 195,8; p <0,01). Las asociaciones de ambos indicadores con las covariables de la línea basal (Tabla 1) justifican un análisis de sobrevida multivariante ajustado por edad y el resto de los factores de riesgo. El 
Tabla 2. D ensidad de incidencia de mortalidad en cohorte de participantes del proyecto San Francisco luego de un seguimiento promedio de 8 años (7.255 años-persona)

\begin{tabular}{|lcccc|}
\hline & \multicolumn{2}{c|}{ Nivel socioeconómico $<10$ puntos } & \multicolumn{2}{c|}{ Educación $<8$ años } \\
& DI Mortalidad* & IC $95 \%$ & DI Mortalidad* & IC 95\% \\
\hline Expuestos & 11,2 & $10,2-12,7$ & 13,5 & $12,4-14,6$ \\
No expuestos & 3,9 & $3,5-4,5$ & 2,3 & $1,9-2,6$ \\
RDI** $^{*}$ & 2,9 & $2,7-3,1$ & 6,0 & $5,8-6,2$ \\
\hline
\end{tabular}

" DI $=$ Densidad de incidencia $\mathrm{x} 1000$ años-persona.

*** Razón de densidades de incidencia, IC 95\% RDI $=\mathrm{e}^{\ln \mathrm{RDI} \pm 1,96 \times \sqrt{ } 1 / \text { eventos expuestos }+1 / \text { eventos no-expuestos. }}$

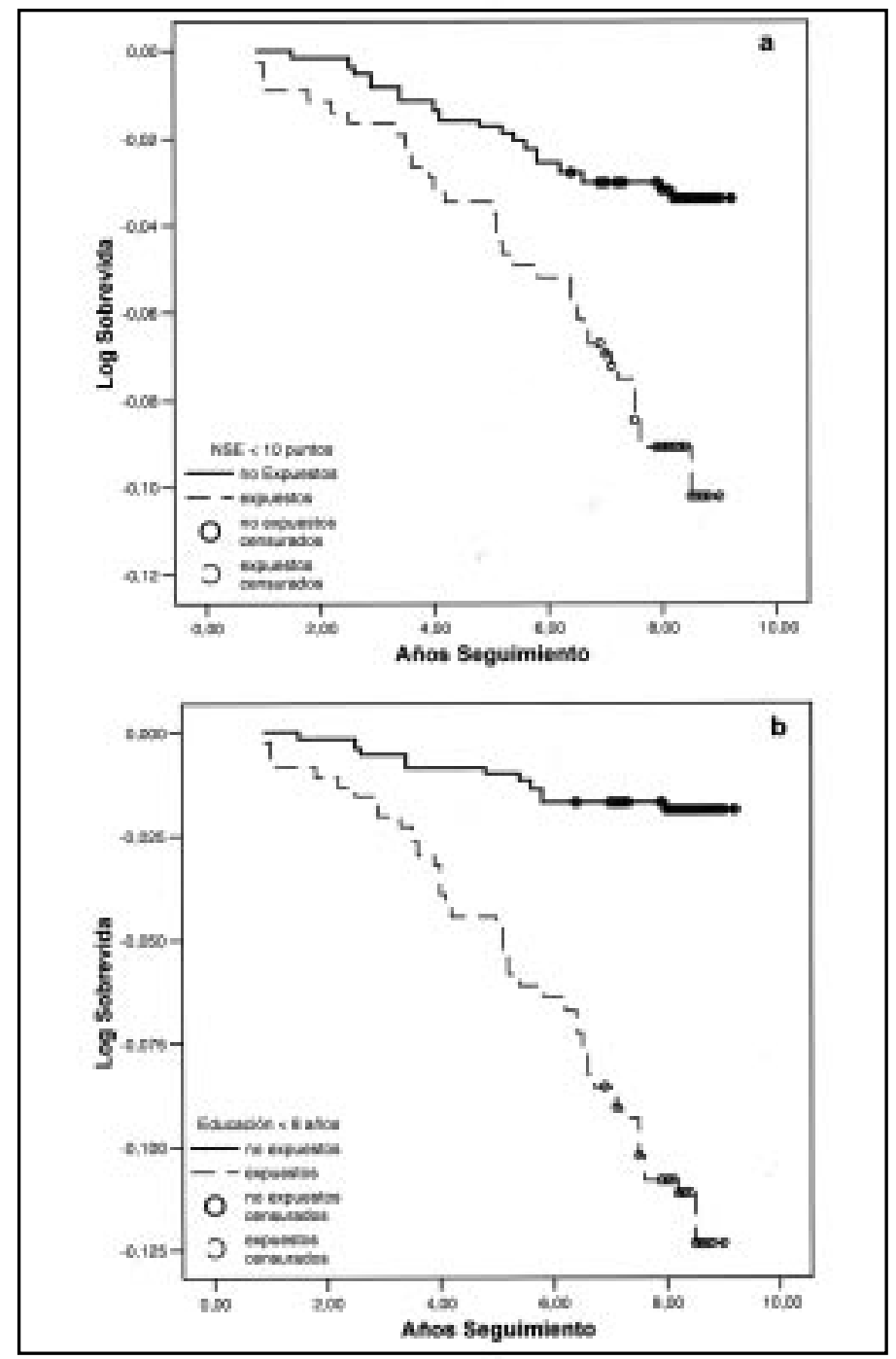

Figura 2. Sobrevida asociada a nivel socioeconómico (NSE) y años de educación en la cohorte de San Francisco de Mostazal, Chile. Curvas de Kaplan-Meier para NSE dicotomizado en 10 puntos (a) y educación alcanzada dicotomizada en 8 años de instrucción primaria (b). La función de sobrevida se presenta en escala logarítmica. riesgo estimado en el modelo de regresión de Cox se presenta en la Tabla 3. No se apreció efecto del sexo, sin embargo el riesgo disminuye notablemente al ajustar por edad y por otros factores de riesgo. Para todos los HRs se observó mayor fuerza de asociación para bajo NEd. Finalmente, las curvas de sobrevida ajustadas por la función multivariante, se presentan en la Figura 3 corroborando menor sobrevida en la cohorte con bajo NSE y bajo NEd. La nosuperposición de las curvas ajustadas, indica que la relación es independiente del efecto de las covariables consideradas en el modelo de regresión de Cox.

\section{DisCuSIÓN}

De acuerdo con el nuevo paradigma de la epidemiología popperiana, los estudios observacionales prospectivos poseen una mayor capacidad para refutar hipótesis causales que los estudios transversales o de caso-control, pues cumplen con la premisa de lógica temporal que va prospectivamente de la exposición simultánea de varios factores de riesgo hasta, el desenlace investigado ${ }^{39}$. Así, esta investigación establece que un NEd inferior al básico ( $<8$ años) está impactando en forma desfavorable, tanto en la magnitud, como en la velocidad del gradiente de mortalidad en la cohorte del PSF. Dada la gran desigualdad social existente en Chile, estos hallazgos no son sorprendentes, 
Tabla 3. Riesgo de muerte por cualquier causa asociado a nivel socioeconómico y nivel de educación estimado a partir de modelo de regresión de $\mathrm{C}$ ox en cuatro bloques aditivos de covariables

\begin{tabular}{|lcccc|}
\hline \multirow{2}{*}{ Bloques } & \multicolumn{2}{c|}{$\begin{array}{c}\text { Nivel socioeconómico }<10 \text { puntos } \\
\text { Hazard Ratio }\end{array}$} & IC $95 \%$ & \multicolumn{2}{c|}{ Hazacación <8 años } \\
& & & & \\
Crudo & $3,34^{*}$ & $2,88-3,87$ & $6,05^{*}$ & $5,05-7,26$ \\
Ajustado por sexo & $3,35^{*}$ & $2,89-3,89$ & $6,08^{*}$ & $5,07-7,31$ \\
Ajustado por edad & $1,33^{*}$ & $1,14-1,26$ & $1,56^{*}$ & $1,28-1,90$ \\
Multivariante & $1,23^{* *}$ & $1,04-1,43$ & $1,54^{*}$ & $1,23-1,85$ \\
\hline
\end{tabular}

†Ajuste simultáneo por edad, sexo, HTA, diabetes mellitus tipo II, dislipidemia criterio ATP III [ref 32], obesidad abdominal (razón cintura/estatura), fumador diario, consumo de alcohol (test de EBBA+) y antecedentes familiares de muerte por evento cardiovascular.

${ }^{*} \mathrm{p}<0,01 ;{ }^{* *} \mathrm{p}<0,05$.
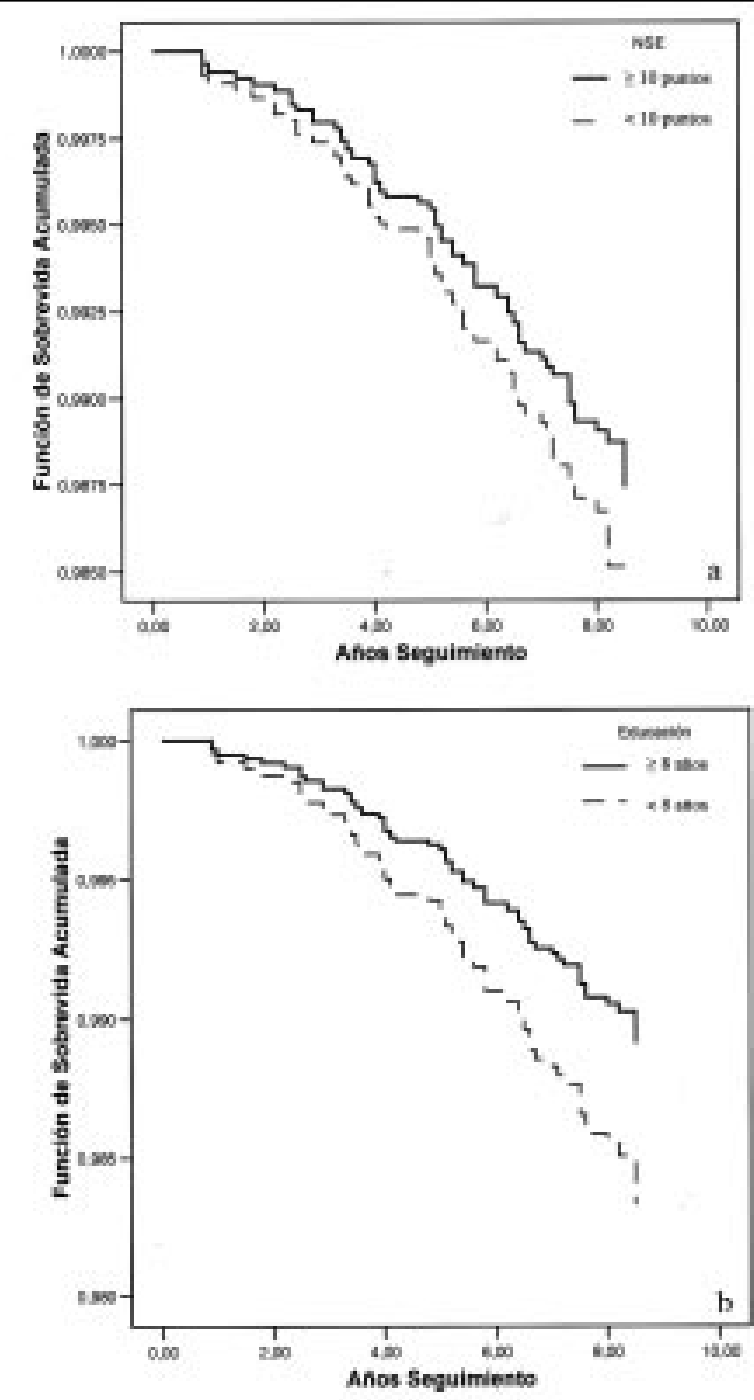

Figura 3. Curvas de sobrevida basadas en el modelo de regresión de riesgos proporcionales de Cox de acuerdo a nivel socioeconómiCo (NSE) dicotomizado en 10 puntos (a) y educación dicotomizada en 8 años de instrucción primaria (b), cohorte de San Francisco de Mostazal, Chile. La función de sobrevida fue ajustada por las covariables de sexo, edad, hipertensión arterial, diabetes mellitus, dislipidemia, tabaquismo, obesidad abdominal, consumo de alcohol y antecedentes familiares de muerte por enfermedad cardiovascular. 
sino biológicamente plausibles y congruentes con el conocimiento epidemiológico disponible. Recientemente, Steenland et al en un estudio longitudinal con más de dos millones de participantes y extenso seguimiento (1959-1996) en EE.UU. establecieron una fuerte relación inversa entre nivel de educación y mortalidad general, incluyendo causas específicas como cáncer, enfermedad coronaria, diabetes y enfermedad pulmonar obstructiva crónica ${ }^{40}$. La relación se mantuvo al ajustar por factores de riesgo convencionales. Por otra parte, Loucks et al, en uno de los informes más recientes provenientes de la cohorte de Framingham ${ }^{41}$, muestran que los años de educación alcanzada están inversamente asociados a la presencia de factores de riesgo inflamatorios, aun después del ajuste por factores de riesgo clínicos de enfermedad coronaria, proporcionando una base biológica que explicaría la relación entre posición socioeconómica, desarrollo de enfermedad cardiovascular y mortalidad. Szanton et al revisan lo que se denomina la teoría de la carga alostática, sintetizando varios mecanismos a través de los cuales la privación socioeconómica determina factores de riesgos biológicos inducidos por el estrés, explicando el fuerte impacto de las desigualdades sobre la salud ${ }^{42}$. Pickering ha revisado el tema en relación con las enfermedades cardiovasculares, en especial, con la hipertensión ${ }^{43}$. Factores como inadecuado acceso a los servicios sanitarios, estilos de vida poco saludables, condiciones laborales desfavorables y dificultades culturales para la penetración de estrategias de promoción y prevención en salud, también están relacionados con una menor posición socioeconómica, especialmente con baja educación ${ }^{5,8,28}$.

La escala de NSE utilizada en el PSF exhibe menor fuerza de asociación que el NEd para mortalidad, sugiriendo que la escolaridad constituye un mejor indicador subrogante de la posición socioeconómica ${ }^{40}$ para medir, tanto la asociación con factores de riesgo (e.g. hipertensión, diabetes, dislipidemia y obesidad abdominal), como el riesgo de muerte. Debido a la autocorrelación entre ambos indicadores, hemos preferido realizar un análisis por separado para ambas variables. De hecho, si consideramos tanto NSE y NEd en un mismo modelo de regresión de Cox, el hazard ratio para NSE $<10$ puntos es 0,96 (IC 95\% 0,801,$16 ; \mathrm{p}=0,71$ ) y para NEd $<8$ años es 1,57 (IC $95 \%$
1,25-1,98; $\mathrm{p}<0,001)$, es decir, la relación observada para el NSE se anula. Osler et al, en un estudio de 13.710 mujeres y 12.018 hombres daneses seguidos durante 12,5 años, establecieron que un mayor ingreso económico individual se asocia a menor mortalidad en un modelo de regresión de Cox ajustado por NEd $<8$ años tabaquismo, sedentarismo, obesidad y consumo de alcohol $^{44}$. Recientemente, Albert et al, luego de 10 años de seguimiento en el Women Health Study (WHS), concluyeron que el nivel de educación exhibe una relación dosis-respuesta inversa para eventos cardiovasculares, independientemente de factores de riesgo tradicionales y emergentes, en contraste con lo observado para el ingreso económico ${ }^{45}$. Así, nuestras observaciones del PSF deberán ser contrastadas con un análisis que considere el ingreso económico y la escolaridad por separado, evaluando dosis respuesta.

Aun cuando el sexo femenino presenta mayor desventaja para cualquiera de los dos indicadores de posición socioeconómica, el análisis multivariante no mostró un mayor riesgo de mortalidad para la población femenina. Futuros estudios analizando causas específicas son necesarios para evaluar con precisión diferencias de riesgo por sexo. Es importante señalar también, que la edad es una variable crucial en estudios epidemiológicos de enfermedades crónicas ${ }^{46}$. Como se constató al analizar las medias por grupos de edad, las generaciones más jóvenes poseen claramente mayor número de años de escolaridad formal, lo que es reflejo de un cambio positivo en Chile durante las últimas décadas ${ }^{8}$. De esta manera el riesgo de muerte se reduce sustancialmente al ajustar por edad, es decir, una parte considerable, pero no la totalidad del riesgo asociado a una baja educación, se explica por el proceso de envejecimiento. Es razonable pensar también, que la mayor mortalidad se debería a la fuerte relación entre poseer bajo NEd y presentar factores de riesgo como HTA, diabetes, dislipidemia, obesidad abdominal, consumo excesivo de alcohol y mayores antecedentes hereditarios de muerte por enfermedad cardiovascular. Sin embargo, luego del ajuste multivariante, la relación es independiente de estos factores, observándose $54 \%$ mayor riesgo en la cohorte con menos de 8 años de escolaridad y $23 \%$ mayor riesgo en la cohorte con NSE menor a 10 puntos. 
De acuerdo a Kawachi et $\mathrm{al}^{47}$, el concepto de desigualdad de salud (heath inequality) es un término genérico utilizado para designar diferencias y variaciones en la salud de individuos y grupos que no implica un juicio de orden moral o político. Whitehead ${ }^{48}$ señala que el concepto de inequidad de salud (health inequity) se refiere a aquellas desigualdades que generan impacto en la salud siendo innecesarias, evitables y que implican una situación de injusticia social. Así, la equidad de salud es un concepto ético, en estrecha relación con los derechos humanos 3,48 . En concordancia con Braveman y Gruskin ${ }^{49}$, las desigualdades sociales que generan disparidades sistemáticas en la salud de una población con diferentes grados de ventaja/desventaja, subyacente a condiciones como educación, riqueza, poder o prestigio, provocan una situación de inequidad de salud cuantificable a escala epidemiológica.

Los resultados presentados aportan datos epidemiológicos concretos que corroboran la hipótesis de la desigualdad como determinante de mortalidad según el nivel de educación alcanzado o posición socioeconómica. En su interpretación positiva, la relación causa-efecto independiente, establecida en una sociedad en transición económica como la chilena, proporciona soporte para afirmar que medidas destinadas a mejorar la educación pueden tener un gran impacto en la salud y sobrevida de la población más vulnerable a través de estrategias poblacionales ${ }^{50}$. Recientemente se

\section{REFERENCIAS}

1. JONG-WOOK L. Global health improvement and WHO: shaping the future. Lancet 2003; 362 : 2083-8.

2. GWATKIN DR. Health inequalities and the health of the poor: what do we know? What can we do? Bull World Health Organ 2000; 78: 3-18.

3. Pan American Health Organization (PaHO). The search for equity: annual report of the director, 1995. Washington, DC: PAHO, 1996.

4. United Nations Development Programme. Human development report 2003: millennium development. New York, NY: Oxford University Press, 2003.

5. Ruger JP, Kim H-J. Global health inequalities: an han publicado resultados de la intervención comunitaria Brookline Early Education Project (BEEP). Luego de 25 años de seguimiento, los adultos jóvenes que fueron intervenidos a través de una estrategia educativa temprana, dirigida a familias y niños hasta la entrada al kindergarten, lograron más altos niveles académicos, ingresos económicos, salud y bienestar, comparados con adultos jóvenes de la población control ${ }^{51}$. En Chile, las estrategias educativas evaluadas por Berríos et al en varias escuelas públicas, aparecen como una intervención eficaz y costo efectiva ${ }^{52}$ cuya implementación y desarrollo por el Estado chileno podría rendir grandes frutos para la salud de la población.

\section{Agradecimientos}

El proyecto San Francisco es patrocinado por Fundación Araucaria, con sede en San Diego, California. Elard Koch y Carlos Díaz son apoyados por beca doctoral MECESUP UCH-0219, Escuela de Salud Pública, Facultad de Medicina, Universidad de Chile. Elard Koch ha sido también apoyado para esta investigación por un grant de Fundación Araucaria. Agradecemos a todos los participantes de esta cohorte y al personal del consultorio San Francisco; a Roberto Lazerini, representante de Laboratorio Labomed. Expresamos nuestro reconocimiento a la Sociedad Chilena de Cardiología por su constante estímulo para el desarrollo de la ciencia epidemiológica en nuestro país.

international comparison. J Epidemiol Community Health 2006; 60: 928-36.

6. Programa de la Naciones Unidas para el Desarrollo (PNUD). Informe sobre Desarrollo Humano 2005. Madrid: Grupo Mundi-Prensa, 2005.

7. Torche F. Unequal but fluid: social mobility in Chile in comparative perspective. Am Sociological Rev 2005; 70: 422-50.

8. Torche F, Wormald G. Estratificación y movilidad social en Chile: entre la adscripción y el logro. CEPAL, Serie Políticas Sociales № 98. Santiago: Naciones Unidas, 2004.

9. Preston SH. The changing relation between mortality and level of economic development. Population Studies 1975; 29: 231-48. 
10. Rodgers GB. Income and inequality as determinants of mortality: an international cross-section analysis. Population Studies 1979; 33: 343-51 (Reprint in Int J Epidemiol 2002; 31: 533-38).

11. Chittleborough CR, Baum FE, Taylor AW, Hiller JE. A life-course approach to measuring socioeconomic position in population health surveillance systems. J Epidemiol Community Health 2006; 60: 981-92.

12. Melchior M, Berkman LF, Kawachi I, Krieger N, Zins $\mathrm{M}$, Bonenfant $\mathrm{S}$ et aL. Lifelong socioeconomic trajectory and premature mortality (35-65 years) in France: findings from the GAZEL cohort study. J Epidemiol Community Health 2006; 60: 937-44.

13. Qureshl AI, Suri MF, SAAD M, Hopkins LN. Educational attainment and risk of stroke and myocardial infarction. Med Sci Monit 2003; 9: CR466-73.

14. Bopp M, Minder C. Mortality by education in German speaking Switzerland, 1990-1997: results from the Swiss National Cohort. Int J Epidemiol 2003; 32: 346-54.

15. Backlund E, Sorlie P, Johnson N. A comparison of the relationships of education and income with mortality: the national longitudinal mortality study. Soc Sci Med 1999; 49: 1373-84.

16. Bucher H, Ragland DR. Socioeconomic indicators and mortality from coronary heart disease and cancer: a 22-year follow-up of middle-aged men. Am J Public Health 1995; 85: 1231-6.

17. Howard G, Anderson R, Russell G, Howard VJ, Burke GL. Race, socioeconomic status, and cause-specific mortality. Ann Epidemiol 2000; 10: 214-23.

18. Sorlie P, Backlund R, Keller J. US mortality by economic, demographic, and social characteristics: the National Longitudinal Mortality Study. Am J Public Health 1995; 85: 949-56.

19. Cubbin C, Gordon S. Socioeconomic inequalities in injury: critical issues in design and analysis. Annu Rev Public Health 2002; 23: 349-75.

20. Donoso E. Reducción de la mortalidad materna en Chile de 1990 a 2000. Rev Panam Salud Pública 2004; 15: 326-30.

21. Szot J. La transición demográfico epidemiológica en Chile, 1960-2001. Rev Esp Salud Pública 2003; 77: 605-13.

22. Szot J. Mortalidad por infarto agudo al miocardio en Chile en el período 1990-2001. Rev Méd Chil 2004; 132: 1227-33.

23. Ministerio de Salud, Gobierno de Chile. Primera Encuesta Nacional de Salud. Informe Final, Mi- nisterio de Salud (MINSAL), Santiago, 2004. Disponible en: http://www.minsal.cl/ici/destacados/ Folleto\%20FINAL.pdf.

24. Sánchez H, Albala C. Desigualdades en salud: mortalidad del adulto en comunas del Gran Santiago. Rev Méd Chil 2004; 132: 453-60.

25. Donoso E. Desigualdad en la mortalidad infantil entre las comunas de la provincia de Santiago. Rev Méd Chil 2004; 132: 461-6.

26. Koch E, Silva C, Manríquez L, Ahumada C. Proyecto San Francisco parte I: alta prevalencia de factores de riesgo cardiovascular en población adulta mayor de 15 años Rev Chil Cardiol 2000; 19: 27-42.

27. Jadue L, Vega J, Escobar MC, Delgado I, Garrido C, Lastra P ET al. Factores de riesgo de enfermedades no transmisibles: método y resultados del programa CARMEN. Rev Méd Chil 1999; 127: 1004-13.

28. Subramanian S, Delgado I, Jadue L, Vega J, Kawachi I. Income inequality and health: multilevel analysis of Chilean communities. J Epidemiol Community Health 2003; 57: 844-8.

29. Косh E, Romero T, Otárola A, Kirschbaum A, Manríquez L, Paredes M. Desigualdad, inequidad y factores de riesgo cardiovascular en Chile: un desafío pendiente. Rev Chil Cardiol 2005; 24: 2809.

30. Koch E, Otárola A, Manríquez L, Kirschbaum A, Paredes M. Predictores de eventos cardiovasculares no fatales en una población urbana en Chile: Experiencia de seguimiento en Proyecto San Francisco. Rev Méd Chil 2005; 133: 1002-12.

31. Chobanian AV, Bakris GL, Black HR, Cushman WC, Green LA, Izzo JL et al. The Seventh Report of the Joint National Committee on Prevention, Detection, Evaluation, and Treatment of High Blood Pressure: the JNC 7 report. JAMA 2003; 289: 2560-72.

32. Expert Panel on Detection, Evaluation, and Treatment of High Blood Cholesterol in Adults. Executive Summary of the Third Report of The National Cholesterol Education Program (NCEP) Expert Panel on Detection, Evaluation, And Treatment of High Blood Cholesterol In Adults (Adult Treatment Panel III). JAMA 2001; 285: 2486-97.

33. EXPert Committee on the Diagnosis and Classification of Diabetes Melutus. Report of the Expert Committee on the Diagnosis and Classification of Diabetes Mellitus. Diabetes Care 1997; 20: 1183-97.

34. Ministerio de SAlud, Gobierno de Chile. Manual: Examen preventivo del adulto. Ministerio de Salud, Santiago, julio 1995. 
35. Koch E, Otárola A, Romero T, Manríquez L, Paredes M, Kirschbaum A et AL. Mediciones antropométricas y riesgo de sufrir un evento cardiovascular no fatal en población chilena. Resultados del proyecto San Francisco. Rev Chil Cardiol 2006; 25: 173-84.

36. Koch E, Díaz C, Romero T, Manríquez L, Paredes M, Kirschbaum A et al. Definiendo puntos de corte antropométricos óptimos para estimaciones de riesgo en la cohorte de San Francisco: asociación con factores de riesgo cardiovascular. Rev Chil Cardiol 2006; 25: 57-8.

37. Barroso-Utra I, Canizares-Pérez M, Lera-Marqués L. Influencia de la estructura de los datos en la selección de los métodos de análisis estadísticos. Rev Esp Salud Pública 2002; 76: 95-103.

38. Otárola A, Kirschbaum A, Manríquez L, Koch E, Bizama MP, Paredes M. Factores de riesgo cardiovascular en población chilena: estudio comparativo Proyecto San Francisco 1999 y Encuesta Nacional de Salud 2003. Rev Chil Cardiol 2004; 23: 251.

39. Косh E, Otárola A, Romero T, Kirschbaum A, Ortúzar E. Popperian epidemiology and the logic of bi-conditional modus tollens arguments for rafutational analysis of randomized controlled trials. Med Hypotheses 2006; 67: 980-8.

40. Steenland K, Henley J, Thun M. All-cause and cause-specific death rates by educational status for two million people in two American Cancer Society cohorts, 1959-1996. Am J Epidemiol 2002; 156: 11-21.

41. Loucks EB, Sullivan LM, Hayes LJ, D’Agostino RB SR, Larson MG, Vasan RS et aL. Association of educational level with inflammatory markers in the Framingham Offspring Study. Am J Epidemiol 2006; 163: 622-8.

42. Szanton S, Gill J, Allen J. Allostatic load: a mechanism of socioeconomic health disparities Biol Res Nur 2005; 7: 7-15.
43. Pickering T. Cardiovascular pathways: socioeconomic status and stress effects on hypertension and cardiovascular function. Ann NY Acad Sci 1999; 896: 262-77.

44. Osler M, Prescott E, Gronback M, Christensen U, Due P, Engholm G. Income inequality, individual income, and mortality in Danish adults: analysis of pooled data from two cohort studies. BMJ 2002; 324: 13-6.

45. Albert MA, Glynn RJ, Buring J, Ridker PM. Impact of traditional and novel risk factors on the relationship between socieconomic status and incident cardiovascular events. Circulation 2006; 114: 2619-26.

46. Lakatta EG, Levy D. Arterial and cardiac aging: Major shareholder in cardiovascular disease enterprises. Part II: The aging health in health: Links to heart disease. Circulation 2003; 107: 346-54.

47. Kawachi I, Subramanian SV, Almeida-Filho N. A glossary for health inequalities. J Epidemiol Community Health 2002; 56: 647-52.

48. Whitehead M. The concepts and principles of equity and health. Int J Health Serv 1992; 22: 429-45.

49. Braveman P, Gruskin S. Defining equity in health. J Epidemiol Community Health 2003; 57: 254-8.

50. Nissinen A, Berríos X, Puska P. Communitybased noncommunicable disease interventions: lessons from developed countries for developing ones. Bull World Health Organ. 2001; 79 : 963-70.

51. Palfrey JS, Hauser-Cram P, Bronson MB, Warfield ME, Sirin S, Chan E. The Brookline Early Education Project: a 25-year follow-up study of a family-centered early health and development intervention. Pediatrics 2005; 116: 144-52.

52. Berríos X, Bedregal P, GuZmán B. Costo-efectividad de la promoción de la salud en Chile. Experiencia del programa "Mírame". Rev Méd Chile 2004; 132: 361-70. 